\title{
El cambio a envases sostenibles como estrategia competitiva de las empresas exportadoras. Un enfoque de Economía Circular
}

\section{The adjustment to sustainable packaging as a competitive strategy for exporting companies. A Circular Economy Approach}

\author{
Yanela Mishel Ortiz Tinoco \\ Universidad Técnica de Machala, Machala, Ecuador \\ yortiz2@utmachala.edu.ec \\ (iD https://orcid.org/0000-0003-0794-8745 \\ Jefferson Andrés Espinoza Castillo \\ Universidad Técnica de Machala, Machala, Ecuador \\ jespinoza6@utmachala.edu.ec \\ (D) https://orcid.org/0000-0003-4037-6523 \\ Mayiya Lisbeth González Illescas \\ Universidad Técnica de Machala, Machala, Ecuador \\ mlgonzalez@utmachala.edu.ec \\ (i) https://orcid.org/0000-0002-5219-3807 \\ Luis Pastor Carmenate Fuentes \\ Universidad Técnica de Machala, Machala, Ecuador \\ xlcarmenate@utmachala.edu.ec \\ (D) https://orcid.org/0000-0001-6057-2746
}

Recepción: 25/07/2021 | Aceptación: 01/09/2021 | Publicación: 10/09/2021

Cómo citar (APA, séptima edición):

Ortiz Tinoco, Y. M., Espinoza Castillo, J. A., González Illescas, M. L., y Carmenate Fuentes, L. P. (2021). El cambio a envases sostenibles como estrategia competitiva de las empresas exportadoras. Un enfoque de Economía Circular. INNOVA Research Journal, 6(3), 246-269.

https://doi.org/10.33890/innova.v6.n3.2021.1849

\section{Resumen}

En el ámbito de los mercados internacionales crece el interés por reducir el impacto ambiental de las actividades económicas. Los gobiernos de países desarrollados se van sumando a la definición de marcos regulatorios como un llamado a las industrias a explorar alternativas de producción en 
El cambio a envases sostenibles como estrategia competitiva de las empresas exportadoras. Un enfoque de Economía Circular

el marco de la Economía Circular. En este contexto, las empresas exportadoras se enfrentan a la necesidad de demostrar que pueden gestionar el cambio desde una Economía Lineal hacia un modelo de producción que integra acciones para recuperar, extraer, transformar y usar el material desechado de productos. El uso de envases sostenibles se identifica como una respuesta estratégica de las empresas globales, en aras de cubrir las expectativas de la sociedad y enfrentar las regulaciones de acceso a los mercados internacionales, a la vez que generan alternativas para mejorar su competitividad. Este artículo tiene por objetivo caracterizar la estrategia de cambio a envases sostenibles realizado por empresas exportadoras, mediante el método de estudio de casos aplicado a una selección de 10 empresas internacionales. Las fuentes de información corresponden a los informes de sostenibilidad y sitios web de las empresas objeto de estudio. Los principales hallazgos señalan que las exportadoras están empleando envases sostenibles como estrategia de adaptación, diferenciación e innovación. Finalmente, se concluye que la implementación del uso de envases biodegradables favorece la reputación de las empresas y el acceso de la oferta exportable a mercados exigentes.

Palabras claves: industria competitiva; envase ecológico; economía circular; normativa legal; estudio de casos.

\begin{abstract}
At present time, the interest in reducing the environmental impact of economic activities is growing in international markets. The governments of developed countries are accompanying the explanation of regulatory frameworks as a call for industries to explore production alternatives within the framework of the Circular Economy. In this respect, exporting companies are faced to the need to demonstrate that they can produce changes using discarded material from a Linear Economy towards a production model harmonizing actions to rescue, obtain and convert. The use of sustainable packaging is identified as a strategic goal by global companies, in order to scope the expectations of society and face the regulations of access to international markets, while generating alternatives to improve their competitiveness. The purpose of this article is to characterize the strategy of change to sustainable packaging carried out by exporting companies, through the case study method applied to a selection of 10 international companies. The information sources fit to the sustainability reports and websites of the companies under study. The main findings indicate that exporters are using sustainable packaging as a strategy for adaptation, differentiation and innovation. To conclude, the implementation of the use of biodegradable packaging benefits the reputation of the companies and the access of the demanding markets.
\end{abstract}

Keywords: competitive industry; ecological packaging; circular economy; legal regulations; case study.

\title{
Introducción
}

El acelerado deterioro ambiental y su influencia en el desarrollo económico, son una preocupación constante a nivel mundial. Diversos grupos de interés han contribuido a generar cambios en las reglas del mercado, en varios países se cuenta con el apoyo de sus gobiernos, a tal punto que se han planteado la llamada "estrategia de Economía Circular".

Con relación a lo expuesto, y bajo el afán de preservar los recursos del planeta, existen varias medidas implementadas que son muestra de ejemplo a seguir por el resto de naciones. Así, en Europa se destaca la Ley de Cambio Climático y Transición Energética, la Agenda 2050 
alineada al Acuerdo de París. La Comisión Europea aplica a la estrategia sobre el uso de los plásticos en una economía circular (Comisión Europea, 2018). En un caso específico como el de España, se han plateado la "reducción de emisiones de gases de efecto invernadero a 2030 en un $20 \%$ respecto a los niveles de 1990" (Ley No 8447, 2021). El gigante asiático dispone de la Ley de Promoción de la Economía Circular de la República Popular de China (CEPAL, 2020).

En América, se identifican varias iniciativas a favor del medio ambiente. México tiene la propuesta de la Ley general de Economía Circular (Senado de México, 2019). En Costa Rica se propuso el Plan de Descarbonización para el período 2018-2050 (Gobierno de Costa Rica, 2018). Países como El Salvador en 2020 y Uruguay en 2019, aprobaron la Ley general de gestión de Residuos.

En este contexto, las empresas exportadoras enfrentan enormes retos respecto a la necesidad de demostrar que pueden gestionar el cambio desde una economía lineal basada en el consumo sin creación de valor en los materiales empleados, a una economía circular que prevé un modelo de producción sostenible. Entre varios aspectos que atañen a las empresas proveedoras de productos a nivel mundial, la producción y gestión de envases de plástico se encuentra en el centro de las discusiones relativas a las prácticas y sustentabilidad de los modelos de producción que emplean. Al hilo de lo expuesto, se evidencia una participación más activa de los consumidores quienes reflejan mayor presión a las empresas sobre su responsabilidad con la sociedad y el medio ambiente (Parrales et al. 2021).

En el ámbito académico se puede resaltar el interés por investigar y analizar la decisión de compra de productos con envases sostenibles a diferencia del convencional (Magnier et al., 2016), bajo un modelo sostenible del desarrollo económico, social y ambiental (Sandoval et al., 2017) con visión en la industria alimentaria para la aplicación de envases amigables con el ambiente (Andrade et al., 2018), iniciativas de reemplazo de los envases tradicionales por ecológicos para comercializar sus productos y brindar seguridad (Debeaufort, 2021)..

Al hilo de lo expuesto desde esta investigación se plantea el objetivo de caracterizar la estrategia de cambio a envases sostenibles realizado por empresas exportadoras, mediante el método de estudio de casos aplicado a una selección de 10 empresas internacionales. En este orden de ideas, destacando la importancia del enfoque de Economía Circular como práctica de las empresas para generar ventajas competitivas en un entorno que exige compromiso con la sociedad y el medio ambiente, se orienta la investigación bajo las interrogantes ¿qué tipo de acciones han implementado las empresas exportadoras, asociadas a la gestión de envases sostenibles en el marco de Economía Circular?, ¿se identifica la estrategia de cambio a envases sostenibles con una mejoría en la competitividad de las empresas?.

La estructura del documento comprende la introducción donde se justifica la importancia del tema, objetivos, preguntas de investigación, seguido del marco teórico en el cual se recogen referencias sobre la Economía Lineal y Economía Circular, estrategia, las implicaciones de los envases en el contexto de las empresas. En metodología se describe y justifica el tipo de investigación, los métodos empleados: estudio de casos, analítico sintético, inductivo deductivo. En el apartado de resultados mediante el desarrollo del estudio de casos se sistematizan variables 
El cambio a envases sostenibles como estrategia competitiva de las empresas exportadoras. Un enfoque de Economía Circular

asociadas al cambio de envases enfocados en las exigencias del mercado. Finalmente, en conclusiones se plantea una síntesis de los argumentos para el cambio a envases sostenibles y su repercusión en la competitividad de las empresas. Adicionalmente se identifican limitaciones y futuras líneas de investigación.

\section{Marco teórico}

\section{Una aproximación a la Economía Lineal y la Economía Circular}

En la actualidad el modelo de producción identificado con la Economía Lineal es el centro de fuertes críticas, entre otras cosas porque muchas empresas no demuestran tener conciencia sobre la afectación que provocan en el medio ambiente (Díaz-Calle et al., 2020), en algunos casos no brindan un tratamiento adecuado a los residuos de los envases (Morocho, 2018), contribuyendo a la pérdida de los ecosistemas (Martínez y Porcelli, 2019).

Con un enfoque integrador, Blomsmaa y Tennant (2020) señalan que la Economía Circular se presenta como una herramienta enfocada en el estudio de los sistemas circulares, donde se prioriza la innovación en el diseño de materiales y la generación de valor alternativo en los residuos de la industria. Representa un modelo de producción donde se valoran los recursos, uso de energía, las estrategias para la gestión de los residuos (Oblitas et al., 2019) y medidas para disminuir la contaminación del medio ambiente.

Con el propósito de proporcionar la visión de varios autores, en la tabla 1 se recogen definiciones contemporáneas sobre Economía Lineal y Economía Circular.

\section{Tabla 1}

Nociones sobre Economía Lineal y Economía Circular

\begin{tabular}{|c|c|c|c|}
\hline Autor & Economía Lineal & Autor & Economía Circular \\
\hline $\begin{array}{l}\text { Ellen } \\
\text { Macarthur } \\
\text { Foundation } \\
\text { (2013) }\end{array}$ & $\begin{array}{l}\text { Describe los límites del actual } \\
\text { sistema de "toma, fabrica y } \\
\text { elimina" y evalúa los riesgos que } \\
\text { plantea para el crecimiento } \\
\text { económico mundial. }\end{array}$ & $\begin{array}{l}\text { Ellen } \\
\text { Macarthur } \\
\text { Foundation } \\
(2013)\end{array}$ & $\begin{array}{l}\text { Es un sistema industrial, } \\
\text { restaurador por intención } \\
\text { y diseño cuyo objetivo es } \\
\text { eliminar los residuos. }\end{array}$ \\
\hline Hidalgo (2017) & $\begin{array}{l}\text { Se relaciona con el crecimiento } \\
\text { económico en la actualidad, de } \\
\text { actividades que se cumplen en las } \\
\text { industrias en un mediano y largo } \\
\text { plazo y los efectos que causan en } \\
\text { el medioambiente. }\end{array}$ & $\begin{array}{l}\text { Prieto et al. } \\
(2017)\end{array}$ & $\begin{array}{l}\text { Relevante sobre los } \\
\text { productos sostenibles, } \\
\text { corresponden a los } \\
\text { modelos y estrategias que } \\
\text { adoptan en parte de la } \\
\text { Economía Circular, para } \\
\text { poder extraer, } \\
\text { transformar, usar y } \\
\text { recuperar recursos ya } \\
\text { obtenidos. }\end{array}$ \\
\hline
\end{tabular}




\begin{tabular}{|c|c|c|c|}
\hline Autor & Economía Lineal & Autor & Economía Circular \\
\hline $\begin{array}{l}\text { Porcelli et al. } \\
(2018)\end{array}$ & $\begin{array}{l}\text { Se refiere a la utilización de } \\
\text { insumos con el uso de recursos } \\
\text { energías fósiles naturales, } \\
\text { provocando un cambio } \\
\text { perjudicial al ecosistema. }\end{array}$ & $\begin{array}{l}\text { Korhonen et } \\
\text { al. (2018) }\end{array}$ & $\begin{array}{l}\text { Se forma de la acción de } \\
\text { mecanismos, que facilitan } \\
\text { la promoción de } \\
\text { estrategias adoptadas a la } \\
\text { producción sostenible y } \\
\text { de las consecuencias a } \\
\text { futuro. }\end{array}$ \\
\hline Arroyo (2018) & $\begin{array}{l}\text { Es un modelo creado como un } \\
\text { sistema de producción lineal, del } \\
\text { consumo de recursos naturales } \\
\text { que provocan escases a futuro. }\end{array}$ & $\begin{array}{l}\text { Arroyo et al. } \\
(2018)\end{array}$ & $\begin{array}{l}\text { En la economía circular } \\
\text { adopta medidas para } \\
\text { disponer de las empresas, } \\
\text { que trabajen con el uso } \\
\text { del desarrollo sostenible, } \\
\text { desde un sentido crítico } \\
\text { ambiental. }\end{array}$ \\
\hline $\begin{array}{l}\text { Balwada et al. } \\
\text { (2021) }\end{array}$ & $\begin{array}{l}\text { Comprende el proceso de tomar, } \\
\text { fabricar y desechar, utilizando sin } \\
\text { control una cantidad de recursos } \\
\text { perjudicial para el ambiente. }\end{array}$ & $\begin{array}{l}\text { Su et al. } \\
(2021)\end{array}$ & $\begin{array}{l}\text { Brinda una oportunidad } \\
\text { como estrategia de mejora } \\
\text { para el cambio de las } \\
\text { actividades de una } \\
\text { sociedad, afectando } \\
\text { escenarios que no } \\
\text { apliquen estas medidas. }\end{array}$ \\
\hline
\end{tabular}

Fuente: Elaboración propia a partir de los autores citados

De acuerdo a los autores de la revisión, la Economía Lineal se identifica con las acciones de "tomar-fabricar-disponer" de grandes cantidades de recursos y energía, que no toma en cuenta el aprovisionamiento para las futuras generaciones. A pesar del resultado negativo al entorno, el modelo de producción lineal se aplica en la industria, lo que constituye un riesgo al bienestar de la humanidad.

La Economía Circular se identifica como un modelo de producción cíclico en el uso, preservación y reutilización de las materias primas y recursos que brinda la naturaleza. Las empresas que logran adaptar este sistema, generan valor agregado a sus productos, a diferencia de otras empresas que usan recursos para crear productos de vida útil baja, que fomenta la recompra y el desecho, sin un proceso de reciclaje (Wilts, 2017).

\section{Iniciativas legales alrededor de la EC, los envases y su influencia en la oferta exportable}

La preocupación por los efectos de las actividades económicas en el medio ambiente hace visible el fortalecimiento de grupos de consumidores que demuestran una mayor concientización por el bienestar común. Las empresas se han visto presionadas a definir estrategias para responder a los cambios que exigen los mercados globales en relación a la Economía Circular. No obstante, de la fuerza que ejercen los consumidores y las empresas, son las definiciones concretas a nivel de 
El cambio a envases sostenibles como estrategia competitiva de las empresas exportadoras. Un enfoque de Economía Circular

gobierno las que marcan pautas de actuación para las industrias proveedoras a nivel global. Tomando como referente los estudios de la CEPAL (2020), en la tabla 2 se exponen las agendas adoptadas por varios países latinoamericanos en materia de Economía Circular.

\section{Tabla 2}

Agendas de economía circular de América Latina y el Caribe

\begin{tabular}{ll}
\hline País & Estrategia \\
\hline Colombia & $\begin{array}{l}\text { Estrategia Nacional de Economía Circular } \\
(2019, \text { aprobada). }\end{array}$ \\
\hline Ecuador & $\begin{array}{l}\text { Pacto por la Economía Circular (2019, } \\
\text { aprobado) } \\
\text { Libro blanco de Economía Circular, hacia } \\
\text { una Estrategia de Economía Circular } \\
\text { (publicado mayo 2021). }\end{array}$ \\
\hline Perú & $\begin{array}{l}\text { Hoja de ruta hacia una Economía Circular } \\
\text { en el sector industrial, (2020, aprobada). }\end{array}$ \\
\hline Costa Rica & $\begin{array}{l}\text { Varias acciones estratégicas de la Política } \\
\text { Nacional de Producción y Consumo } \\
\text { contemplan aspectos de EC. }\end{array}$ \\
\hline Brasil, Chile, & $\begin{array}{l}\text { Plan de acción en Economía Circular } \\
\text { (aprobado 2019). }\end{array}$ \\
Uruguay &
\end{tabular}

Fuente: CEPAL (2020).

La existencia de las mencionadas agendas revela la incorporación de la Economía Circular a las prioridades de la política pública en América Latina y el Caribe. Se evidencian planes que promueven acciones estratégicas en sectores como la agricultura y el comercio. En este sentido, las empresas exportadoras pueden considerar como referente de futuras actuaciones las especificaciones de las agendas de Economía Circular, debido a que revisten un impacto en la gestión del flujo de materiales, envases y empaques con los que se ofertan sus productos.

Explorando lo que acontece a nivel de la Unión Europea, se destaca la Ley de Cambio Climático y Transición Energética que muestra una serie de objetivos alineados a favor del medio mediante los "Planes Nacionales de Energía y Clima (PNIEC)" (Ley No 8447, 2021). Además, la existencia de la "estrategia a largo plazo 2050" crea un marco legal de gobernanza para reforzar la participación y compromiso de los países pertenecientes.

La Economía Circular tiene varias dimensiones, no obstante, su meta principal es residuos cero. Así, para las empresas exportadoras el cambio a envases sostenibles en sus productos se identifica como una necesidad frente a las exigencias de las normativas que reflejan la urgencia de implementar modelos de producción responsables con el medio ambiente. 
Un ejemplo de la presión que se ejerce actualmente al respecto del uso de plásticos se puede ejemplificar en la Directiva SUP (UE) 2019/904, con base jurídica en el artículo 192 del Tratado de Funcionamiento de la UE, que trata sobre políticas medio ambientales para prevenir y disminuir residuos de plástico en el mar, que se originan por su único uso. En efecto, el marco normativo incide en la acción que realiza cada país, empresa y cliente, en un mercado con miras al cambio climático (Lozano Cutanda y Poveda, 2019), que a raíz de la Directiva 2018/851UE prohíbe el ingreso de plásticos de un solo uso para alimentos, habiéndose hecho efectiva el 3 de julio del 2021.

De forma complementaria, la Directiva (UE) 2019/904 abarca distintas alternativas para trasformar el consumo de productos plásticos de un solo uso frente a la disponibilidad de opciones sostenibles. En la Unión Europea existen medidas de sensibilización a los consumidores para impulsar acciones responsables, con el propósito de disminuir el uso de plástico convencional. Así, se prohíbe mediante ley, el uso de envases o cajas para alimentos que no requieren elaboración como, frutas, verduras, y ensaladas.

La Unión Europea con el Pacto verde europeo propone lograr la descarbonización del sistema para el 2050, con medidas hacia el diseño de bienes sostenibles, de la cadena de valor en puntos estratégicos como: alimentos, envases, embalajes, plásticos y programas relacionados a la materia.

Al hilo de las ideas expuestas, existen otras iniciativas que reflejan el interés por mitigar el impacto ambiental de los plásticos. Así, la red denominada El Pacto del Plástico del Reino Unido de la Fundación Ellen MacArthur, siendo una entidad que organiza a participantes a nivel nacional o regional como gobiernos, empresas y consumidores con el fin de realizar proyectos con visión común e intercambiar prácticas responsables hacia una Economía Circular. La red en mención tiene como participantes de la Unión Europea a los países de: Francia, Portugal, Polonia, Holanda, además de Estados Unidos, Canadá, Australia, Nueva Zelanda, Chile, y Países Bajos (Ellen Macarthur Foundation [EMF], 2017).

\section{Caracterización de envases sostenible}

Los envases ecológicos tienen una relación con los principios del desarrollo sostenible referido a lo económico, social y ambiental. Acciones como consumir, desechar, y contaminar los recursos son efectos producidos por las industrias, siendo un desafío la creación de envases sostenibles para una mejora de los impactos ambientales (Boz et al., 2020).

La creación de envases biodegradables se considera una práctica indispensable en el desarrollo sostenible, con la particularidad que se distingue de envases comunes por la reducción de los recursos y desperdicios generados durante su ciclo de vida (Lindh et al., 2016).

De acuerdo a Ma y Moultrie (2017), la implementación de propuestas para el desarrollo de envases ecoamigables parte del diseño, que ayuda a contrarrestar la utilización de otros envases alejados de los principios de sostenibilidad. El almidón se considera una fuente de envase sostenible proveniente de la naturaleza. Para Gadhave et al. (2018), los envases de materiales 
El cambio a envases sostenibles como estrategia competitiva de las empresas exportadoras. Un enfoque de Economía Circular

derivados del petróleo, han generado complicación en relación al desarrollo sostenible. El uso de almidón se emplea como materia verde de polímeros, que forman películas biodegradables evitando desechos de envases para alimentos.

Como expresan Yaradoddi et al. (2020), usar materiales biodegradables para envases incrementa el desarrollo de componentes orgánicos como carboximetilcelulosa obtenida de desechos agrícolas, que ofrece protección al producto y la descomposición del recipiente, lo cual genera un valor ambiental.

Existen diferentes organizaciones que buscan establecer criterios y mejoras sobre el envasado sostenible. Así, la Organización Europea para el envasado y el medio ambiente "EUROPEN", con sede en Bruselas-Bélgica, tiene el propósito de consolidar un mercado europeo con la utilización de envases para la protección del producto, logrando recopilar e informar datos, decisiones y opciones para promover la seguridad ambiental, sujetos a políticas establecidas por la Unión Europea.

La organización fundada en 1993 está conformada por 62 miembros corporativos y 11 asociaciones nacionales, permite el acceso a toda empresa con el objetivo de consolidar sus operaciones con un rendimiento económico y sostenible, dirigido a la innovación de los envases (European Organization for Packaging and the Environment [EUROPEN], 2021). Está gobernada por un consejo, asignando participación a cada uno de los miembros y regulado por un comité a cargo de un Director Gerente que administra la Secretaria de la EUROPEN, el comité ejecutivo está representado por socios de las empresas de Coca-Cola, Tetra Pak, Marte, Hacerse un nido, Danone, PepsiCo, Dow Europa, Unilever.

La Coalición de Envases Sostenibles por sus siglas en inglés "SPC" ubicada en Charlottesville-Estados Unidos es otra organización encargada de incentivar a la innovación y capacitación continua de sus miembros, fue creado en 2005 dirigida al manejo de los envases mediante proyectos o acciones que fomentan una educación a la sostenibilidad. Está conformado por toda empresa, institución y organización gubernamental que busca incorporar y promover a sus operaciones el uso de empaques sostenibles (Sustainable Packaging Coalition [SPC], 2021). Con la mira de mantener una participación equitativa de todos los representantes se designa un Comité Ejecutivo conformado por dos fabricantes de materiales, dos marcas empresariales y dos industrias fabricantes de envases para garantizar el cumplimiento de los objetivos. El comité se encuentra representado por empresas como PepsiCo, KoolEarth solutions, Amcor, Unilever, Printpack, Danone North América, 3M.

Otra organización que destaca es la Alianza de envases sostenibles (SPA) con base en Australia, ofrece herramientas y estrategias relacionadas con la sostenibilidad de envases permitiendo beneficios económicos y ambientales. Dispone de una herramienta de evaluación empresarial que analiza el impacto ambiental de los envases dirigidos a industrias, empresas de embalajes para lograr la sostenibilidad (Sustainable Packaging Alliance [SPA], 2021).

A manera de sistematización, en la tabla 3 se recoge una síntesis sobre los criterios de envasado sostenible referidos por las tres organizaciones descritas. 


\section{Tabla 3}

Escuelas de criterios de envasado sostenible

\begin{tabular}{ll}
\hline $\begin{array}{l}\text { Organización Europea } \\
\text { para el }\end{array}$ & $\begin{array}{l}\text { El empaque debe diseñarse con el fin de cumplir } \\
\text { con los criterios propios de su creación, y } \\
\text { después del uso reciclarse o recuperarse para } \\
\text { Ambiente } \\
\text { (EUROPEN) }\end{array}$ \\
\hline $\begin{array}{l}\text { disminuir el impacto ambiental. } \\
\text { soalición de envases }\end{array}$ & $\begin{array}{l}\text { Los envases al usar tecnología de producción } \\
\text { limpia, aseguran la inocuidad en alimentos } \\
\text { durante el ciclo de vida. }\end{array}$ \\
\hline & $\begin{array}{l}\text { El envase debe ser efectivo, eficiente y limpio. } \\
\text { Al basarse en el ambiento social, económico, } \\
\text { haciendo más con menos y contener materiales } \\
\text { no contaminantes. }\end{array}$ \\
\hline
\end{tabular}

Fuente: (Rezaei et al., 2018).

Las organizaciones describen un criterio sobre las acciones en el uso de envases sostenibles, comprende las actividades como parte de los recursos que ocupan los envases que se emplean de los recursos naturales y están definidos y publicados por cada una de las instituciones. Las operaciones de las empresas deben ser reguladas mediante un registro de actividades, como parte de los criterios a evaluar en el diseño de envases, y determinar si un producto puede considerarse sostenible.

\section{La estrategia en el cambio a envases sostenibles}

Las empresas cumplen un rol protagónico en las actividades económicas alrededor del mundo, siendo las decisiones que adoptan con relación a la protección del ambiente, una respuesta estratégica a la sociedad respecto a su compromiso con el desarrollo sostenible (Yngfalk, 2019).

Recurriendo a la visión de autores clásicos, la estrategia se define como un conjunto de acciones combinadas con recursos (Ohmae, 1989). Según Nicolai Foss se diseña sobre la base de un diagnóstico interno y externo para formular respuestas pertinentes a la dinámica del entorno (Andrews, 1971, Capitulo 5). La estrategia debe encajar con la información del contexto (mercado en el que compiten las empresas exportadoras), así, las empresas deben emplear su capacidad para adaptarse rápidamente a las presiones externas derivadas del interés por la sostenibilidad ambiental (Reuter, 2010).

En orden a las ideas expuestas, la sustitución de envases de plástico por envases sostenibles se identifica como una estrategia de diferenciación que permite a las empresas exportadoras cubrir las expectativas de los grupos de actores interesados para mantener una posición competitiva frente a otras empresas. En este sentido, la adopción de envases sostenible se asocia con las estrategias de adaptación (Zeriti et al., 2014 y Zhao et al., 2014) y de diferenciación (Hah y Freeman, 2014), 
El cambio a envases sostenibles como estrategia competitiva de las empresas exportadoras. Un enfoque de Economía Circular

ya que las empresas exportadoras se están situando en una ruta de actuación de respeto al medio ambiente como forma de diferenciarse y dar respuesta a las presiones de la sociedad a nivel global.

Frente a la importancia de las repercusiones en las actividades económicas en el medio ambiente, la competitividad de las empresas exportadoras se encuentra ligada a su capacidad para optimizar los recursos y disponer de ellos de una forma adecuada (Lewandowsk, 2016). La incorporación de la innovación para una gestión sostenible de los envases permite incrementar las posibilidades de aceptación frente al consumidor y a la vez potencializar el producto o la marca en el mercado (Carreño y Baquero, 2019).

Para Chamorro (2019), las empresas son parte indispensable de los procesos de desarrollo sustentable, y lo reflejan cuando resuelven problemas medioambientales y crean oportunidades de negocios sostenibles y crecimiento económico.

En Colombia la empresa Alpina ha desarrollado un marco de innovación como base de la estrategia para ser pionera en el mercado de la industria alimentaria, integrando los requisitos del consumidor sostenible y la legislación en las transformaciones de sus procesos bajo la premisa de alimentar saludablemente y sin daños al entorno (González, 2013). El compromiso de Alpina se materializa a través del ecodiseño, elaboración de envases y empaques que son producto de material reciclado, así también, sorbetes biodegradables, etiquetas de cartón, generando valor e innovación. Además, el propósito de ser plástico neutro para el 2022, lo que conlleva el retiro de plástico que ubica en el mercado cada año (Alpina, 2020).

El caso de una microempresa italiana que llegó a ser líder en Europa para el manejo del envasado a granel seguro y sostenible, logrando competitividad en el mercado con una estrategia de innovación en el desarrollo de un sistema diferente a la competencia (Lombardi et al., 2019). El sistema implementado por la empresa Eceplast, proporcionar un manejo eficiente de los recursos reduciendo los residuos y su impacto en el medio ambiente.

En Ecuador la empresa El Ordeño, respondiendo a una sociedad que se encuentra en el auge de la concientización ambiental, emplea bagazo de caña de azúcar para la fabricación de sus envases de cartón (El Ordeño, 2019). Desde el año 2019 ha realizado adaptaciones en su modelo de producción, lo cual le ha permitido consolidarse en el camino de la sostenibilidad que promueve el bienestar en toda la cadena de valor.

La gestión de las empresas se está orientando a la sostenibilidad, marcando una diferencia de valor agregado en el mercado y desarrollando una ventaja competitiva en relación a otras empresas, (Pigatto et al., 2019).

La reducción de desechos, la optimizan de recursos y el uso de materiales biodegradable en los envases, se ha posicionado como una oportunidad para cubrir las exigencias de los consumidores a nivel internacional (Mahalik y Nambiar, 2021). Así también, se busca el crecimiento sobre la competencia de otras empresas, priorizando la productividad con seguridad en la utilización de envases sostenibles, además del impacto favorable de imagen corporativa ante la sociedad. 


\section{Metodología}

El presente artículo se circunscribe en una investigación de tipo documental, empleando un enfoque cualitativo, de alcance descriptivo para caracterizar el uso de envases sostenibles que utilizan las empresas exportadoras. Según Sampieri et al. (2014), la investigación cualitativa, adopta un planteamiento de problema específico, el cual se refleja en la exposición realizada alrededor de la necesidad de las empresas de responder a las demandas de los grupos de interés globales que exigen visibilidad del compromiso ambiental y social en la ejecución de las actividades económicas.

Se utilizaron los métodos teóricos como el analítico sintético, inductivo deductivo, para el análisis e interpretación de lo expresado por los autores seleccionados en la revisión de la literatura, que permitió identificar la economía circular como estrategia de diferenciación, caracterización del desarrollo sostenible en envases, marco legal y su influencia en la oferta exportable.

Para la presentación de los resultados se consideraron dos fases. En la primera fase se empleó el método de estudio de casos bajo el criterio de Walter Sahel en 1977. A partir de un muestreo no probabilístico se seleccionaron 10 empresas que dan cuenta de cambios en la gestión de envases para la oferta de sus productos en los mercados internacionales. Las fuentes empleadas para la selección corresponden a informes de sostenibilidad de varias entidades como Fundación EU-LAC (2018), Danone (2018), Grupo Arcor (2017), páginas webs de las empresas.

Para la recopilación de datos de los 10 casos, se analizaron los reportes e informes de sostenibilidad de cada empresa, enfocando la atención en las estrategias empleadas, la evaluación de desempeño en la gestión de cambio dirigida a la sostenibilidad y protección del medio ambiente. Las empresas se identifican con un elevado nivel de participación e incidencia en los mercados internacionales, así: Danone, Tetra Pak, Unilever, Henkel y Eceplast; en América Latina: Arcor y Nestlé. Además, se consideraron tres empresas del territorio ecuatoriano que tienen participación en mercados extranjeros y ocupan en sus productos envases sostenibles, y poseen certificaciones internacionales.

Estas empresas son: Pacari, con reconocimiento de "Empresa B" que aplica un modelo de gestión sostenible y de Certificación World Fair Trade; Perla Chocolate Organic cuenta con Denominación de Origen Cacao Arriba, BPM para las Buenas Prácticas de Manufactura, Certificación Orgánica para Europa y Estados Unidos de Norte América; la Fabril posee un documento que garantiza el Sistema de Seguridad Alimentaria 22000, Análisis de Peligros y Puntos de Control Críticos (HACCP) y Calidad ISO 9001, respaldadas con premios y certificaciones internacionales al cuidado del medio ambiente.

En una segunda fase de la presentación de resultados se adopta como referencia la investigación realizada por Rezaei et al. (2018) quienes seleccionan criterios para el diseño de envases sostenibles, considerando las necesidades de los actores de la cadena de suministros de alimentos y alternativas presentes en el mercado de proveedores. Así también los estudios de Mura et al. (2019), aplicados a 254 Pymes italianas, indican la importancia del uso de los envases ecológicos como estrategia competitiva a nivel mundial y criterios para su diseño. Estos autores 
El cambio a envases sostenibles como estrategia competitiva de las empresas exportadoras. Un enfoque de Economía Circular

coinciden en señalar como dimensiones asociadas a los envases sostenibles las variables: social, ambiental y económica. Adicionalmente se realizan adaptaciones del trabajo desarrollado por Ahmad y Wong (2019), quienes aplicando el modelo Delphi definen criterios de sustentabilidad asociados a los envases de la industria alimentaria en Malasia.

Finalmente, los resultados se sistematizaron en una matriz de variables que integran las observaciones de las 10 empresas consideradas como casos de estudio: información (3 subvariables), economía (5 subvariables), ambiental (5 subvariables), social (4 subvariables).

\section{Resultados y Discusión}

De acuerdo a los procedimientos expuestos en el apartado de metodología, en una primera fase se recogen datos relevantes de cada empresa objeto de análisis: descripción de sus productos, envase ecológico que aplica y el impacto social de estas medidas.

\section{Caso 1}

Unilever de origen británico-neerlandesa fundada en 1929, dedicada a la higiene, hogar y alimentos. Para el 2010 la empresa impulsó el Plan de Vida Sustentable que considera tres principios: optimizar la salud de las personas; minimizar el impacto ambiental; y calidad de vida. Durante el Plan de Sustentabilidad, han logrado reducir costos y riesgos, lo cual ha potenciado la cadena de valor un negocio que promueva la Economía Circular. El grupo de desarrollo sostenible de la empresa es responsable de elaborar el informe anual de sostenibilidad, que evalúa el rendimiento del Plan con respecto a los principios de los Objetivos del Desarrollo del Milenio y sobre los compromisos internacionales relacionados al impacto ambiental (Unilever, 2010).

Unilever se ha planteado como objetivo la transición de los envases plásticos a reciclables y reutilizables para el año 2025. Una muestra para el cumplimiento de este objetivo es la creación del producto Cif desarrollado mediante una nueva tecnología que permite a los consumidores rellenar y reusar los envases del concentrado de producto, se añade agua y su mezcla finaliza en la comodidad del domicilio, teniendo como resultado la versión original del producto de limpieza. En efecto, cambia el patrón de consumo y aporta al propósito empresarial, de generar valor con acciones responsables ambientalmente.

\section{Caso 2}

Desde 1995, la empresa líder europea Eceplast ha desarrollado soluciones innovadoras en el sector alimentario, automotriz, químico, y medioambiental para las actividades de empacar, almacenar y realizar envíos bajo estricto control ambiental. Además, implementa buenas prácticas con normativas internacionales como ISO 9001: 2015; ISO 14001: 2015; ISO 22000: 2005. Los tipos de embalaje son utilizados para los productos a granel o secos que protegen la carga a factores externos. Están creados para disminuir costos y reducir la huella de carbono, bajo proyección para aplicar el reciclaje y reutilización (Eceplast, 2021).

Desde 2008 hasta la actualidad, la empresa se encuentra en una fase denominada industria 4.0, caracterizada por la automatización tecnológica de sus procesos. Además de la planta de 
producción, el sitio alberga oficinas administrativas y comerciales, un sector de investigación, desarrollo y control de calidad

\section{Caso 3}

Arcor es un grupo multinacional de origen argentino, creado en 1951, dedicado a la fabricación y distribución de alimentos de consumo masivo, agro negocios y envases. Con la estrategia de gestión sustentable desarrolla marcas que permiten una apertura en más de 100 países. El informe de sustentabilidad Arcor 2020 está elaborado por Global Reporting Initiative "GRI", entidad encargada de mostrar transparencia y asignar responsabilidad a las empresas con temas relacionados a los estándares de sostenibilidad. También se enfatizan los indicadores que permiten una comparativa con años anteriores, logrando evaluar el desempeño de la empresa en lo económico, social y ambiental, relacionado con los estándares de Envasado de Productos Agrícolas, Alimentos Procesados y envases "SASB" (Gerencia Corporativa de Sustentabilidad [GCS], 2020).

La empresa enfrenta desafíos relacionados en la comercialización de productos envasados, por ello busca el desarrollo de empaques que minimicen el impacto ambiental. Arcor está trabajando en la reducción de envases, la reutilización de materiales desechados, el reemplazo de materiales con otros de menor incidencia en el ambiente. El uso de materiales plásticos de esta empresa, ha tenido una reducción del $11 \%$ en los últimos 7 años, el $96 \%$ del papel. Con la implementación de políticas para la reducción y reutilización de envases convencionales se utiliza el cartón que es de origen sustentable.

\section{Caso 4}

Tetra Pack es una empresa que inició sus operaciones en el año 1943, con la premisa de convertirse en líder mundial de soluciones para envasados y procesamiento de alimentos. Disponible en más de 160 países ofrece productos seguros y eco amigables con el ambiente. La visión de la compañía es proteger los alimentos, hacer que estos sean seguros y accesibles en cualquier territorio. Mantener un liderazgo responsable y sostenible sobre el envasado y manipulación en productos lácteos, bebidas, comidas entre otros alimentos perecibles.

La empresa presenta en su Reporte de sustentabilidad 2020 temas referentes a los desafíos que debe enfrentar desde la perspectiva social para lograr sostenibilidad. Una de sus acciones destacables es el suministro de materiales reutilizables en las plantas de fabricación de envasados para ser distribuidos en todo el mundo (Tetra Pak, 2020).

Actualmente ha aumentado la producción de envases y contenidos renovables ofertados por la empresa con la elaboración de sorbetes de papel, capas y tapas de plásticos a base de caña de azúcar. Fomenta la circularidad, eliminando residuos y empleando polímeros reciclados a los envases, asegurando el uso de un 10\% de plástico reciclado en las bebidas distribuidas en Europa.

\section{Caso 5}


La empresa Henkel surge en el año 1876 en Alemania. Desde sus inicios se enfoca en la mejora, adoptando en sus procesos el desarrollo ambiental y social, lograr conseguir un impacto positivo de los productos y servicios tecnológicos que ofrecen la mayoría de países. La empresa está comprometida en cumplir con esos objetivos basados en el concepto de economía circular y usar envases 100\% reciclables y reutilizables hasta el 2025.

Henkel en su Reporte de Sostenibilidad 2019 señala el compromiso de la organización por desarrollar estrategias dirigidas al manejo responsable de los recursos. Se apoya en la implementación de los Objetivos del Desarrollo Sostenible, incorporados por los Estados Miembro de las Naciones Unidas en 2015 junto a la participación de las industrias y la cadena de valor de productos (Henkel AG y Co. KGaA, 2019).

Para Henkel los envases sostenibles ayudan a proteger los productos, brindar información sobre su uso y la responsabilidad medioambiental, el problema radica en su consumo, una vez usados se desechan y pasan a convertirse en residuos. Entre las operaciones que emprendió la empresa se destaca la fabricación de tubos de pasta de dientes reciclables. Además, los productos de limpieza ocupados en botellas, están siendo elaborados por material $100 \%$ reciclado, brindando una protección adecuada al producto, satisfacción al cliente y ofreciendo una contribución al planeta.

\section{Caso 6}

En 1944 grupo Nestlé apertura en Colombia, la empresa Ciccolac, constituida junto a Borden Inc. Simultáneamente, conforma la Industria Nacional de Productos Alimenticios (INPA S.A), en la distribución de comestibles y bebidas, con una cartera de productos nacionales e internacionales. Su fin es mejorar la calidad de vida y brindar un futuro saludable, que contribuya a la calidad de alimentos para la sociedad.

La empresa se ha propuesto reducir el impacto en el ambiente, usando empaques reciclables y reutilizables en el $100 \%$ de sus productos para el 2025, para disminuir el uso de 162 toneladas de plásticos. En Colombia la meta consiste en recolectar para el año 2021 cerca de 3400 toneladas de materiales en productos desechados mediante proyectos de reaprovechamiento.

Colombia a través de Nestlé, será el país precursor en implementar fundas de papel para proteger los sorbetes en los productos de la marca Milo y Klim, como parte de la visión estratégica de reemplazar las cajas por papel, se dejará de usar cerca de diez millones de sorbetes y bolsas plásticas en el país, como se menciona en el informe de compromiso de Nestlé con el apoyo de instituciones que apoyan el desarrollo de envases reutilizables (Rever, 2019).

\section{Caso 7}

La Fabril es una empresa ecuatoriana creada que inició sus actividades en el año 1968. Se enfoca en la innovación de sus productos con los más altos estándares de calidad, en el marco de protocolos de Responsabilidad Social y Sustentabilidad. Está presente en mercado nacional e internacional, dedicado al ámbito de alimentos, cuidado del hogar y personal. La gama de 
productos industriales es: sucedáneos de chocolate; grasas para heladería; frituras; galletas y confites.

Para el cumplimiento del objetivo empresarial La Fabril desarrolla tecnología innovadora en las técnicas de operatividad, para disminuir el impacto ambiental. Una de las acciones fue añadir a la producción de margarina el proceso de in mould labelling (IML), lo que significa un empaque ecológico para alimentos (González y González, 2018).

Fabril presenta su Informe de Sostenibilidad basado en los principios de la Norma AA1000 que corresponde a una norma de aseguramiento del esquema innovador a favor del desarrollo sostenible. Orienta a las empresas a determinar procesos ordenados que abarcan las partes interesadas para la creación de estrategias y un sistema de comunicación directo a los miembros corporativos. Estas actividades proveen un esquema a profesionales que elaboran informes de sostenibilidad de la iniciativa de informe global, a fin de lograr resultados ambientales, sociales y económicos (Accountability, 2006).

\section{Caso 8}

La empresa ecuatoriana Perla Organic Chocolate fundada en el año 2017, se encuentra dedicada a la siembra de cacao y producción variada de chocolate libre de químicos y plaguicidas, cumpliendo con los más altos niveles de calidad, cuenta con certificaciones orgánicas (Perla Organic Chocolate, 2020).

Es considerada, precursora en la industria chocolatera del Ecuador en adoptar la transición de una economía lineal a circular, bajo los tres principios $3 \mathrm{R}$ (reducir, recuperar y reciclar). En efecto ha sido galardonada con International Chocolate Awards en 2018 y Premios Latinoamérica Verde en 2020.

Perla Organic Chocolate cuenta con empaques biodegradables, elaborados con semillas recicladas. Como resultado es el papel plantable, a disposición del consumidor lo que genera una conexión entre el productor, cliente y el planeta que se beneficia con soluciones de tecnología limpia.

\section{Caso 9}

Pacari es una empresa chocolatera que inicia sus actividades en el año 2002 y con seis años de trabajo empezó sus exportaciones a varios mercados a nivel mundial. Pacari es un término quechua que traducido al español significa "naturaleza", promulga el vínculo entre empresa, sociedad y ambiente, siendo parte del proceso cuyo fin es el cambio de la cultura de consumo.

Ha recibido múltiples reconocimientos que incluye más de 200 premios a nivel nacional e internacional. Se destaca por su producción orgánica afianzada en operaciones de sostenibilidad en el marco de los protocolos que define el sello Demeter Biodynamic Certification que valida la producción de materia prima sin químicos y los procesos en la cadena de valor bajo un entorno regenerativo, cuidando el ecosistema (Noboa, 2017). 
La responsabilidad frente a la comunidad y el medio ambiente se refleja en la línea de productos a los clientes, la cual no culmina hasta su consumo. En este sentido, Pacari se propuso a la transición en el sistema de empaques, ya que reconoce la incidencia del plástico el sector empresarial (Pacari, 2021).

Su empaque es creado a base de celulosa vegetal que sustituye el material plástico, da como resultado su degradación en 180 días en lugar de 180 años y así contribuye a disminuir el impacto en el medio ambiente.

\section{Caso 10}

Danone es una empresa francesa fundada en el año1919 con cuatro líneas de producción: nutrición médica e infantil, agua mineral, leches fermentadas y artículos de origen vegetal. Esta empresa está aplicando un modelo de producción sustentado en la Economía Circular, promoviendo la fabricación de los envases con proceso innovadores y materiales reciclados. Se ha planteado el reto de transformar todos los envases convencionales a ecológicos para el año 2025 en varios países de la Unión Europea.

Con la política de envases sostenibles, apertura fuentes de empleo a favor del medio ambiente, como inversiones para ejecutar acciones que logren cuidar los recursos naturales que son agotables. Una práctica que no afecta la calidad del producto es el ecodiseño para envases.

El grupo Danone en su Informe de Estrategia de Sostenibilidad 2018 destaca que mantiene alianzas con la Fundación Ellen MacArthur, y el Programa de las Naciones Unidas para el Medio Ambiente con el propósito de establecer un compromiso general para tratar la contaminación y el incremento de desechos plásticos. Además, en conjunto con Nestlé, PepsiCo y Origin Materials ha lanzado al mercado un envase con el $75 \%$ de origen biológico, y se propone para el año 2025 emplear el 100\% de componentes orgánicos (Danone, 2018).

Para tratar la segunda etapa de los resultados, en línea con el objetivo y pregunta de investigación, se expone la sistematización de resultados en la tabla 4 a partir de las variables: información, economía, ambiental, social (Rezaei et al., 2018; Ahmad y Wong, 2019; Mura et al., 2019). La información se ha obtenido a partir de información identificada en los reportes de sostenibilidad de las empresas y sus páginas webs.

\section{Tabla 4}

Análisis de variables de los casos de estudio

\begin{tabular}{|c|c|c|c|c|c|c|c|c|c|c|c|}
\hline \multicolumn{2}{|r|}{ Casos } & $\begin{array}{c}\text { Caso } \\
1\end{array}$ & $\begin{array}{c}\text { Caso } \\
2\end{array}$ & $\begin{array}{c}\text { Caso } \\
3\end{array}$ & $\begin{array}{c}\text { Caso } \\
4\end{array}$ & $\begin{array}{c}\text { Caso } \\
5\end{array}$ & $\begin{array}{c}\text { Caso } \\
6\end{array}$ & $\begin{array}{c}\text { Caso } \\
7\end{array}$ & $\begin{array}{c}\text { Caso } \\
8\end{array}$ & $\begin{array}{c}\text { Caso } \\
9\end{array}$ & $\begin{array}{c}\text { Caso } \\
10\end{array}$ \\
\hline \multirow{3}{*}{ 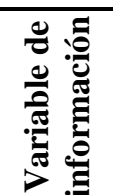 } & $\begin{array}{l}\text { Descripción de la } \\
\text { empresa }\end{array}$ & $\mathrm{X}$ & $X$ & $X$ & $X$ & X & $X$ & $X$ & $X$ & $X$ & $X$ \\
\hline & Descripción de producto & $\bar{X}$ & $\bar{X}$ & $\bar{X}$ & $\bar{X}$ & $\bar{X}$ & $\bar{X}$ & $\bar{X}$ & $\bar{X}$ & $X$ & $\bar{X}$ \\
\hline & Acceso a información & $\mathrm{X}$ & $\mathrm{X}$ & $\mathrm{X}$ & $\mathrm{X}$ & $\mathrm{X}$ & $\mathrm{X}$ & $\mathrm{X}$ & $\mathrm{X}$ & $\mathrm{X}$ & $\mathrm{X}$ \\
\hline
\end{tabular}


Yanela Mishel Ortiz Tinoco, Jefferson Andrés Espinoza Castillo, Mayiya Lisbeth González Illescas y Luis Pastor

ISSN 2477-9024. Innova Research Journal (Septiembre-Diciembre, 2021). Vol. 6, No. 3, pp. 246-269

\begin{tabular}{|c|c|c|c|c|c|c|c|c|c|c|c|c|}
\hline & & Casos & $\begin{array}{c}\text { Caso } \\
1\end{array}$ & $\begin{array}{c}\text { Caso } \\
2\end{array}$ & $\begin{array}{c}\text { Caso } \\
3\end{array}$ & $\begin{array}{c}\text { Caso } \\
4\end{array}$ & $\begin{array}{c}\text { Caso } \\
5\end{array}$ & $\begin{array}{c}\text { Caso } \\
6\end{array}$ & $\begin{array}{c}\text { Caso } \\
7\end{array}$ & $\begin{array}{c}\text { Caso } \\
8\end{array}$ & $\begin{array}{c}\text { Caso } \\
9\end{array}$ & $\begin{array}{c}\text { Caso } \\
10\end{array}$ \\
\hline \multirow{12}{*}{ 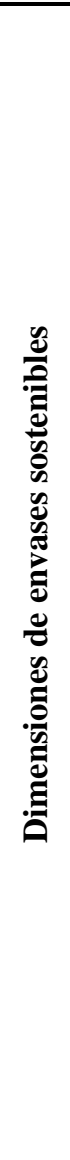 } & \multirow{4}{*}{ 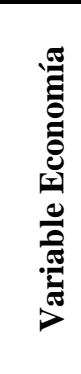 } & $\begin{array}{l}\text { Ingreso al mercado en } \\
\text { pro del medioambiente }\end{array}$ & $X$ & $X$ & $X$ & $X$ & $X$ & $X$ & $X$ & $X$ & $X$ & $X$ \\
\hline & & $\begin{array}{l}\text { Diseñado para ahorro de } \\
\text { materiales de envases }\end{array}$ & $X$ & $X$ & $X$ & $X$ & $X$ & $X$ & $X$ & $X$ & $X$ & $X$ \\
\hline & & $\begin{array}{l}\text { Promueve la } \\
\text { competitividad en } \\
\text { función del envase }\end{array}$ & $X$ & $X$ & $X$ & $X$ & $X$ & $X$ & $X$ & $X$ & $X$ & $X$ \\
\hline & & $\begin{array}{l}\text { Relación costo/beneficio } \\
\text { del envase }\end{array}$ & X & $X$ & $X$ & $X$ & X & $X$ & $X$ & $X$ & $X$ & $X$ \\
\hline & \multirow{4}{*}{ 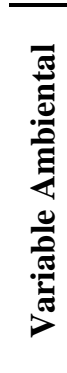 } & $\begin{array}{l}\text { Método de reciclaje, } \\
\text { reutilización y } \\
\text { desechado de envases }\end{array}$ & - & $X$ & $X$ & $X$ & $X$ & $X$ & $X$ & $X$ & - & $X$ \\
\hline & & $\begin{array}{l}\text { Emplea materiales } \\
\text { biodegradables }\end{array}$ & $X$ & - & - & $X$ & - & - & - & $X$ & $\mathrm{X}$ & $X$ \\
\hline & & $\begin{array}{l}\text { Sistema de gestión de } \\
\text { riesgo ambiental }\end{array}$ & $X$ & $X$ & $X$ & $X$ & $X$ & $X$ & $X$ & $X$ & $\mathrm{X}$ & $X$ \\
\hline & & $\begin{array}{l}\text { Disminución del recurso } \\
\text { de transporte }\end{array}$ & $X$ & - & - & - & - & - & - & - & - & - \\
\hline & \multirow{4}{*}{ 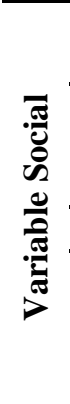 } & $\begin{array}{l}\text { Aporta bienestar para la } \\
\text { comunidad }\end{array}$ & $X$ & X & $X$ & $X$ & $X$ & $X$ & $X$ & $X$ & $\mathrm{X}$ & $X$ \\
\hline & & $\begin{array}{l}\text { Informes de } \\
\text { responsabilidad y } \\
\text { sostenibilidad } \\
\end{array}$ & $X$ & $\mathrm{X}$ & $\mathrm{X}$ & $\mathrm{X}$ & $\mathrm{X}$ & $\mathrm{X}$ & $X$ & - & - & $\mathrm{X}$ \\
\hline & & Inocuidad del producto & $\mathrm{X}$ & $\mathrm{X}$ & $\bar{X}$ & $\bar{X}$ & $\bar{X}$ & $\mathrm{X}$ & $\bar{X}$ & $\bar{X}$ & $\mathrm{X}$ & $\bar{X}$ \\
\hline & & $\begin{array}{l}\text { Adopta las normas y } \\
\text { políticas } \\
\text { gubernamentales e } \\
\text { internacionales }\end{array}$ & $X$ & $X$ & $\mathrm{X}$ & $X$ & $X$ & $X$ & $X$ & $X$ & $X$ & $X$ \\
\hline
\end{tabular}

Fuente: Elaboración propia a partir de los autores citados

De los datos obtenidos de la tabla 4 se destaca la información recopilada a partir de variables y subvariables. Respecto a la variable general de información se refleja que las 10 empresas proporcionan acceso a reportes de sustentabilidad, descripción de la organización y productos, lo cual implica la facilidad y apertura a datos por parte de la sociedad.

En relación a la variable de economía, se identifica que las empresas analizadas han logrado destacarse en los mercados internacionales por sus prácticas organizacionales enmarcadas en el compromiso con el medioambiente, operando con diseños de envases que priorizan el ahorro de materiales, lo que les ha permitiendo ser competitivos en función del producto respecto a la relación costo/beneficio que pueden brindar los envases. Por otro lado, siendo superior el costo de los envases biodegradables, este puede cubrirse ya que los compradores están dispuestos a pagar un precio más alto por el valor agregado que encierra la experiencia de compra de un producto sostenible (Singh y Pandey, 2019).

Las subvariables de la dimensión ambiental se refieren a parámetros que dan cuenta de la gestión ambiental que realizan las empresas. Las 10 empresas en estudio incorporan en sus 
El cambio a envases sostenibles como estrategia competitiva de las empresas exportadoras. Un enfoque de Economía Circular

modelos de producción métodos de reciclaje, reutilización, gestión de desechos de envases, y se enfocan en aprovechamiento de materiales biodegradables y diseños ecoamigables.

En el aspecto social las empresas logran cumplir con los criterios definidos, en el aporte de bienestar para la comunidad siendo transparente con los informes que brindan sobre responsabilidad y sostenibilidad, adoptando las normas y políticas de gobierno interno y externo, así evaluar los riesgos que pueden acarrear relacionado con la inocuidad del producto dirigido a la sociedad.

La tendencia del mundo globalizado compromete al sector empresarial a ser flexible, adaptarse, examinar alternativas ecológicas y realizar cambios e innovación, con la finalidad de ser competitivos (Giraldo y Quintero, 2017). Los empresarios están orientados a la selección de proveedores ecológicos para alcanzar competitividad a nivel global (Ecer, 2020). Estas decisiones en función de la gestión de sostenibilidad, determinan indicadores relacionados a los costos, tiempo de comercialización y las operaciones en el desarrollo de envases biodegradables.

Los hallazgos permiten sustentar que los envases sostenibles inciden en la competitividad de las empresas exportadoras cuyo fin es acceder a los mercados internacionales con propuestas diferenciadoras que contribuyan a generar ventajas competitivas.

\section{Conclusiones}

Los consumidores globales reflejan mayor interés respecto al deterioro ambiental provocado por las empresas de las cuales se proveen. Son más exigentes y lo demuestran con un cambio en sus patrones de consumo. La contaminación provocada por el uso de plástico para la elaboración de los envases y la gestión de los mismos ha motivado a las autoridades gubernamentales de países desarrollados a definir acciones y marcos regulatorios para incentivar el cambio del modelo de producción lineal a un modelo de Economía Circular.

La conservación del medio ambiente y el uso de recursos son elementos que se destacan entre los principios y objetivos de la sostenibilidad. El desarrollo sostenible forma parte de una práctica sustentable, para la creación y utilización de envases ecoamigables, que regulan el uso de materiales convencionales, igualmente fomentan alternativas respecto al diseño, procesos y materia prima para futuras generaciones (Boz, et al., 2020). Como manifestación de las decisiones de ajuste estratégico a las transformaciones del entorno, crece el número de empresas que responden a la presión de la ciudadanía global, mediante la adaptación de sus modelos de producción a la Economía Circular. Así, se observa que las empresas exportadoras se involucran en acciones para recuperar, extraer, transformar y usar el material desechado de productos, evaluando alternativas para emplear materiales derivados de recursos naturales (Blomsmaa y Tennant, 2020).

Las empresas que se suman al cambio de envases sostenibles están siendo coherentes con sus acciones ya que se están preparando para enfrentar las regulaciones internacionales y a la vez que generan alternativas para mejorar su competitividad. Una muestra de la alerta de los mercados se puede ejemplificar en la medida adoptada por la Unión Europea, relativa a la restricción de 
importación de plásticos de un solo uso, que acaba de entrar en vigencia el 3 de julio del presente año 2021 (Lozano-Cutanda y Poveda, 2019).

Por otra parte, existen estudios que sustentan que las empresas productoras de alimentos con mejor posicionamiento a nivel mundial, consideran relevante el empaque ecológico (MolinaBesch y Palsson, 2019), y se encuentran haciendo previsiones para cambios a empaques con eficiencia ambiental. Estas decisiones de carácter estratégico potencian el acceso a mercados estrictos en gestión ambiental, incluso promueven en las industrias la generación de valor agregado que se suma a la actividad comercial (CEPAL, 2020).

A nivel de América Latina existen programas gubernamentales que proporcionan orientaciones a los productores sobre el ajuste necesario de su oferta exportable en el marco de responsabilidad ambiental y social, así se puede verificar en las distintas agendas, hojas de ruta y libros blancos de Economía Circular. En este contexto, el cambio a envases sostenibles tiene el potencial de fomentar las relaciones comerciales con los principales mercados internacionales, a través de productos que no compiten por su precio, sino por una estrategia diferenciadora que se revela en la acción ambiental de las empresas exportadoras.

Este trabajo tiene implicaciones para el sector exportador, toda vez que podrían considerarse las acciones de las empresas internacionales analizadas, como referentes de estrategia competitiva en el marco del modelo de producción de Economía Circular. Coincidiendo con las conclusiones de (Coelho et al, 2020), quienes señalan que la oferta exportable bajo criterios de envases sostenibles, mejora la reputación corporativa, perspectiva del consumidor y acceso a los mercados internacionales más exigentes, desde este trabajo plantea que las empresas aplican la alternativa de envases sostenibles, reflejan un cambio de paradigma en la gestión de procesos coherentes con el desarrollo sostenible, incrementando la competitividad mientras responden a las exigencias en temas sociales y ambientales (Chamorro, 2019).

Finalmente, considerando las limitaciones de la investigación de tipo teórico y descriptivo, basada en datos secundarios, lo cual no permite la generalización de los resultados, se propone como futura línea de investigación el abordaje de la problemática tratada desde un enfoque cuantitativo, con la incorporación de datos primarios y el análisis e identificación de relaciones entre las variables que conforman las dimensiones de envases sostenibles.

\section{Referencia Bibliográfica}

Ahmad, S., y Wong, K. Y. (2019). Development of weighted triple-bottom line sustainability indicators for the Malaysian food manufacturing industry using the Delphi method. Journal of Cleaner Production, 1167-1182. https://doi.org/10.1016/j.jclepro.2019.04.39.

Alpina. (2020). Informe de sostenibilidad 2020. https://sostenibilidadalpina2020.com/

Andrade, B. R., Rivera, M. A., y Guzmán, H. L. (2018). El empaque como oportunidad para el desarrollo del producto y el consumidor responsable; una mirada desde la industria en Norteamérica y Suramérica. Saber, Ciencia y Libertad, 13(1), 164-179. https://dialnet.unirioja.es/descarga/articulo/6571929.pdf 
El cambio a envases sostenibles como estrategia competitiva de las empresas exportadoras. Un enfoque de Economía Circular

Andrews, K. R. (1971). The Concept of Corporate Strategy. En N. J. Foss (Ed.) Resources, Firms, and Strategies: A Reader in the Resource-based Perspective, (1-378). Prensa de la Universidad de Oxford. https://bit.ly/3y $7 \mathrm{cxq} 8$

Arroyo-Morocho, F. R. (2018). La Economía Circular Como Factor De Desarrollo Sustentable Del Sector Productivo. INNOVA Research Journal, 3(12), 78-98. https://doi.org/10.33890/innova.v3.n12.2018.786

Arroyo-Morocho, F. R., Bravo-Donoso, D. N., y Rivera-Valenzuela, M. A. (2018). Economía Circular: Un Camino Hacia Un Quito Más Sostenible. INNOVA Research Journal, 3(11), 139-158. https://doi.org/10.33890/innova.v3.n11.2018.767

Balwada, J., Samaiya, S., y Mishra, R. P. (2021). Packaging Plastic Waste Management for a Circular Economy and Identifying a better Waste Collection System using Analytical Hierarchy Process. 28th CIRP Conference on Life Cycle Engineering. https://doi.org/10.1016/j.procir.2021.01.102

Blomsmaa, F., y Tennant, M. (2020). Circular economy: Preserving materials or products?. Introducing the Resource States framework. Resources, Conservation \& Recycling, 1-13. https://doi.org/10.1016/j.resconrec.2020.104698

Boz, Z., Korhonen, V., y Sand, C. K. (2020). Consumer Considerations for the Implementation of Sustainable Packaging: A Review. Sustainability, 12(6), 1-34. https://doi.org/10.3390/su12062192

Carreño, N. E., y Baquero, Z. Y. (2019). Sostenibilidad como estrategia de competitividad empresarial en sistemas de producción Agropecuaria. Revista Estrategia Organizacional, 8(1), 9-26. https://doi.org/10.22490/25392786.3168

Chamorro, A. A. (2019). La Sustentabilidad de las Pymes comerciales como factor de acometividad. $\quad$ Científica $\quad$ Multidisciplinar, $\quad 3(1), \quad$ 134-155. https://ciencialatina.org/index.php/cienciala/article/view/15/8

Comisión Económica para América Latina y El Caribe (2020). Serie Comercio Internacional, $\mathrm{N}^{\circ}$ 159. El comercio internacional y la economía circular en América Latina y el Caribe. $\mathrm{N}$. Mulder y $\quad$ M. https://www.cepal.org/sites/default/files/publication/files/46618/S2000783_es.pdf

Comisión Europea. (2018). Comunicación de la Comisión al Parlamento Europeo, al Consejo, al Comité Económico y Social Europeo y al Comité de las Regiones. https://eurlex.europa.eu/legal-content/ES/TXT/HTML/?uri=CELEX:52018DC0028\&from=FR

Danone. (2018). Come como piensas. Departamento de General Secretary. https://bit.ly/3zw0m6x

Debeaufort, F. (2021). Active biopackaging produced from by-products and waste from food and marine industries. Open Bio, 11, 984-998. https://doi.org/10.1002/2211-5463.13121

Díaz-Calle, N., Menoscal-Pincay, R., y González-Illescas, M. (2020). Economía Circular: desafíos para una visión estratégica de las empresas exportadoras. Compendium: Cuadernos de $\begin{array}{lllll}\text { Economía } & y & \text { Administración, } & 7(3), & 120-135 .\end{array}$ https://doi.org/10.46677/compendium.v7i3.865

Eceplast. (2021). Eceplast Sustainable Packaging. Obtenido de Liner to Liner: closing the loop on plastic packaging recycling: http://www.eceplast.com/notizie/liner-to-liner-closingthe-loop-on-plastic-packaging-recycling/

Ecer, F. (2020). Multi criteria decision making for green supplier selection using interval type-2 fuzzy AHP: a case study of a home appliance manufacturer. Operational Research. (1-35). https://doi.org/10.1007/s12351-020-00552-y

Esta obra se comparte bajo la licencia Creative Common Atribución-No Comercial 4.0 International (CC BY-NC 4.0) Revista de la Universidad Internacional del Ecuador. URL: https://www.uide.edu.ec/ 
Yanela Mishel Ortiz Tinoco, Jefferson Andrés Espinoza Castillo, Mayiya Lisbeth González Illescas y Luis Pastor Carmenate Fuentes

ISSN 2477-9024. Innova Research Journal (Septiembre-Diciembre, 2021). Vol. 6, No. 3, pp. 246-269

Ellen Macarthur Foundation. (2013). Towards the circular economy. https://www.ellenmacarthurfoundation.org/assets/downloads/publications/TCE_Report2013.pdf

Ellen Macarthur Foundation. (2017). Pacto de plásticos: Una red de iniciativas nacionales o regionales que trabajan hacia una economía circular para los plásticos. https://www.newplasticseconomy.org/projects/plastics-pact

El Ordeño. (2019). Memoria de sostenibilidad 2019. KOMM Comunicación Estratégica. https://elordeno.com/web/Memoria_ELORDENO_2019_final_webRS2.pdf

European Organization for Packaging and the Environment. (22 de junio de 2021). Structure and governance. https://www.europen-packaging.eu/

Fundación EU-LAC. (2018). Estudios de Caso sobre Modelo de Economía Circular e Integración de los Objetivos de Desarrollo Sostenible en Estrategias Empresariales en la UE y la ALC. Scharlau GmbH.

Gadhave, R. V., Das, A., Mahanwar, P. A., y Gadekar, P. T. (2018). Starch Based Bio-Plastics: The Future of Sustainable Packaging. Open Journal of Polymer Chemistry, 8, 21-33. https://doi.org/10.4236/ojpchem.2018.82003

Gerencia Corporativa de Sustentabilidad. (2020). Reporte de sustentabilidad 2020. Gerencia Corporativa de Comunicaciones Institucionales. https://imagen.arcor.com/institucional/reporte-de-sustentabilidad-2020.pdf

Giraldo-Henao, E. G., y Quintero-Sepúlveda, I. C. (2017). Innovación, Consumo y $\begin{array}{llll}\text { Competitividad: } & \text { Apuestas } & \text { Regionales. }\end{array}$ https://revistas.upb.edu.co/index.php/rice/article/view/5384/5040

Gobierno de Costa Rica (2018). Plan de Descarbonización. San José: Gobierno de Costa Rica. https://cambioclimatico.go.cr/wp-content/uploads/2019/02/PLAN.pdf

González, A. D., y González, A. L., (2018). La Fabril Group sustainability Report 2018. Guayaquil: La Fabril S.A. https://www.lafabril.com.ec/wp-content/uploads/2020/04/LaFabril-Memoria-2018-_English-web.pdf

González, J. P. (2013). Alpina: un caso de innovación para la competitividad. Revista de Ingeniería, (38), 78-85. https://www.redalyc.org/pdf/1210/121028408012.pdf

Hah, K. y S. Freeman (2014). Multinational enterprise subsidiaries and their CSR: a conceptual framework of the management of CSR in smaller emerging economies. Journal of Business Ethics, 122, 125-136. https://doi.org/10.1007/s10551-013-1753-8

Henkel, AG. y Co, KGaA. (2019). Sustainability Report 2019. https://www.henkel.com/resource/blob/1038544/af1684b38200328128a80b5dd18e485e/d ata/2019-sustainability-report.pdf

Hidalgo-García, M. (2017). Un nuevo impulso hacia la economía circular. Instituto Español de Estudios Estratégicos. https://dialnet.unirioja.es/descarga/articulo/6231820.pdf

Korhonen, J., Nuur, C., Feldmann, A., y Eshetu-Birkie, S. (2018). Circular economy as an essentially contested concept. Journal of Cleaner Production. 175, 544-552. https://doi.org/10.1016/i.jclepro.2017.12.111

Ley 7 del 2021. Cambio climático y transición energética. 20 de mayo del 2021. BOE-No-20218447. https://www.boe.es/boe/dias/2021/05/21/pdfs/BOE-A-2021-8447.pdf

Lewandowsk, M. (2016). Designing the Business Models for Circular Economy-Towards the Conceptual Framework. Sustainability, 8(43), 2-28. https://doi.org/10.3390/su8010043 
El cambio a envases sostenibles como estrategia competitiva de las empresas exportadoras. Un enfoque de Economía Circular

Lindh, H., William, H., Olsson, A., y Wikström, F. (2016). Elucidating the Indirect Contributions of Packaging to Sustainable Development: A Terminology of Packaging Functions and Features. Package. Technology Science, 29, 225-246. https://doi.org/10.1002/pts.2197

Lombardi, M., Maffia, G., y Tricase, C. (2019). Sustainable Bulk-Packaging System for Sugar Shipping: Case Study of the Enterprise Leader in Europe. Administrative science, 9(91), 216. https://doi.org/10.3390/admsci9040091

Lozano, C. B., y Poveda, P. (2019). Directiva (UE) 2019/904, del Parlamento Europeo y del Consejo, relativa a la reducción de determinados. Gómez-Acebo y Pombo. https://www.actualidadjuridicaambiental.com/wpcontent/uploads/2019/08/2019_09_03_Lozano_Poveda_Directiva-plastico.pdf

Ma, X., y Moultrie, J. (2017). What stops designers from designing sustainable packaging? A review of eco-design tools with regard to packaging design. Engineering, 1-14. https://doi.org/10.1007/978-3-319-57078-5_13

Magnier, L., Schoormans, J., y Mugge, R. (2016). Judging a product by its cover: Packaging sustainability and perceptions of quality in food products. Food Quality and Preference, 53, 132-142. http://doi.org/10.1016/i.foodqual.2016.06.006

Mahalik, N. P., y Nambiar, A. N. (2021). Trends in food packaging and manufacturing systems and technology. Trends in Food Science \& Technology, 21, 117-128. https://doi.org/10.1016/j.tifs.2009.12.006

Martínez, A., y Porcelli, A. (2019). Estudio sobre la economía circular como una alternativa sustentable frente al ocaso de la economía tradicional. Lex, 17(23), 1-40. http://doi.org/10.21503/lex.v17i23.1679

Molina-Besch, K., y Palsson, H. (2019). A simplified environmental evaluation tool for food packaging to support decision-making in packaging development. Packaging Technology and Science, 4(33), 141-157. https://doi.org/10.1002/pts.2484

Morocho, F. R. (2018). La economía circular como factor de desarrollo sustentable del sector $\begin{array}{lllll}\text { productivo. INNOVA Research Journal, } & 3(12), & \text { 78-98. }\end{array}$ https://doi.org/10.33890/innova.v3.n12.2018.786

Mura, M., Longo, M. l., y Zanni, S. (2020). Circular economy in Italian SMEs: A multi-method study. Journal of Cleaner Production, 245, 1-35. https://doi.org/10.1016/j.jclepro.2019.118821

Noboa, A. V. (2017). Pacari: Chocolate Orgánico Premium. Harvard Business Publishing. https://hbsp.harvard.edu/product/BL0003-PDF-ENG

Oblitas, J., Sangay, M., Rojas, E., y Castro, W. (2019). Economía circular en residuos de aparatos eléctricos y electrónicos. Revista de Ciencias Sociales (Ve), 25(4), 1-11. https://www.redalyc.org/jatsRepo/280/28062322016/html/index.htm

Ohmae, K. (1989). La mente del estratega (Primera ed.). México: McGraw Hill.

Pacari. (18 de junio de 2021). Acerca de Pacari, un caso de éxito. https://www.pacari.com/prensa/media-kit/

Parrales-Zumba, C., Trelles-Vera, G., y González-Illescas, M. (2021). La responsabilidad social empresarial y su papel estratégico en la competitividad de las empresas exportadoras internacionales. Yachana Revista Científica, 10(2). http://revistas.ulvr.edu.ec/index.php/yachana/article/view/670

Pigatto, G., Pigatto, G. A., Satolo, E. G., y Negreti, A. d. (2019). The importance and the adaptation of internal resources as a competitive advantage for the internationalization of food 
companies: An analysis of Brazilian companies. Grey Systems: Theory and Application, 9(3), 305-320. https://doi.org/10.1108/GS-10-2018-0048.

Perla Organic Chocolate (2020). Sobre Nosotros. Obtenido de La economía circular que cambiará nuestro futuro: https://perlaorganicchocolate.com/

Porcelli, A. M., y Martínez, A. N. (2018). Análisis legislativo del paradigma de la economía circular. Direito GV. 14(3), 1067-1105. https://doi.org/10.1590/2317-6172201840

Prieto-Sandoval, V., Jaca, C., y Ormazabal, M. (2017). Economía circular: Relación con la evolución del concepto de sostenibilidad y estrategias para su implementación. Memoria Investigaciones en Ingeniería (15),

85-95. http://revistas.um.edu.uy/index.php/ingenieria/article/view/308/366

Reuter, C., K. Foerstl, E. Hartman y C. Blome (2010), Sustainable global supplier management: the role of dynamic capabilities in achieving competitive advantage. Journal of Supply Chain Management, 46(2), 45-63. https://doi.org/10.1111/j.1745-493X.2010.03189.x

Rever, J. (2019). Compromisos Nestlé con la tierra. https://empresa.nestle.es/sites/g/files/pydnoa431/files/2019-11/folleto-compromisosnestle-tierra-def_3.pdf

Rezaei, J., Papakonstantinou, A., Tavasszy, L., Pesch, U., y Kana, A. (2018). Sustainable productpackage design in a food supply chain: A. Packaging Technology and Science, 92. https://doi.org/10.1002/pts.2418

Sampieri, R. H., Collado, C. F., y Lucio, M. d. (2014). Metodología de la Investigación. McGrawHill / Interamericana Editores. http://observatorio.epacartagena.gov.co/wpcontent/uploads/2017/08/metodologia-de-la-investigacion-sexta-edicion.compressed.pdf

Sandoval, V. P., Jaca, C., y Ormazabal, M. (2017). Economía circular relación con la evolución del concepto de sostenibilidad y estrategias para su implementación. Ingeniería. (15), 8595. http://revistas.um.edu.uy/index.php/ingenieria/article/view/308/366

Senado de México (12 de noviembre de 2019), Gaceta: LXIV/2PPO-51/101326. https://www.senado.gob.mx/64/gaceta_del_senado/documento/101326

Singh, G., y Pandey, N. (2019). Revisando los envases ecológicos desde una perspectiva de costos. Benchmarking Int. J., 10. https://doi.org/10.1108/BIJ-04-2018-0099

Su, C. y Urban, F. (2021). Circular economy for clean energy transitions: A new opportunity under the COVID-19 pandemic. Applied Energy. 28, 1-11. https://doi.org/10.1016/j.apenergy.2021.116666

Sustainable Packaging Alliance. (2021). Welcome to SPA. http://www.sustainablepack.org/

Sustainable Packaging Coalition. (2021). Our members. https://sustainablepackaging.org/

Tetra pak. (2020). ENABLING TRANSFORMATION Sustainability Report 2020. Tetra pak group.

https://www.tetrapak.com/content/dam/tetrapak/publicweb/gb/en/sustainability/document s/sustainability-report2020.pdf

Unilever. (2010). Plan Unilever para una vida sostenible: Pequeñas acciones hacen una gran diferencia.

https://www.compromisorse.com/upload/noticias/006/6208/unilevervidasostenible.pdf

Wilts, H. (2017). Key Challenges for Transformations Towards a Circular Economy - The Status Quo in Germany. International Journal of Waste Resources, 7(262), 1-5. https://bit.ly/3zwhN75 
El cambio a envases sostenibles como estrategia competitiva de las empresas exportadoras. Un enfoque de Economía Circular

Yaradoddi, J. S., Banapurmath, N., Ganachari, S. V., Soudagar, M., Mubarak, N., Hallad, S. A., y Hugar, S. (2020). Biodegradable carboxymethyl cellulose based material for sustainable packaging application. Scientific Reports, 10, 1-13. https://doi.org/10.1038/s41598-020$\underline{78912-\mathrm{Z}}$

Yngfalk, C. (2019). Subverting sustainability: market maintenance work and the reproduction of corporate irresponsibility. Journal of Marketing Management, 35(17-18), 1-20. https://doi.org/10.1080/0267257X.2019.1682031

Zeriti, A., M.J. Robson, S. Spyropoulou y C.N. Leonidou (2014). Sustainable export marketing strategy: fit and performance. Journal of International Marketing. 22(4), 44-66. https://doi.org/10.1509\%2Fjim.14.0063

Zhao, M., S.H. Park y N. Zhou (2014). MNC strategy and social adaptation in emerging markets. Journal of International Business Studies. 45, 842-861. https://doi.org/10.1057/jibs.2014.8 